\title{
Data Reduction for an ICT Network in the Smart Grid
}

DOI:

10.1109/IE.2013.26

Link to publication record in Manchester Research Explorer

\section{Citation for published version (APA):}

Pourmirza, Z., \& Brooke, J. M. (2013). Data Reduction for an ICT Network in the Smart Grid. In Proceedings - 9th International Conference on Intelligent Environments, IE 2013/Proc. - Int. Conf. Intelligent Environ., IE (pp. 224227). IEEE Computer Society . https://doi.org/10.1109/IE.2013.26

\section{Published in:}

Proceedings - 9th International Conference on Intelligent Environments, IE 2013|Proc. - Int. Conf. Intelligent Environ., IE

\section{Citing this paper}

Please note that where the full-text provided on Manchester Research Explorer is the Author Accepted Manuscript or Proof version this may differ from the final Published version. If citing, it is advised that you check and use the publisher's definitive version.

\section{General rights}

Copyright and moral rights for the publications made accessible in the Research Explorer are retained by the authors and/or other copyright owners and it is a condition of accessing publications that users recognise and abide by the legal requirements associated with these rights.

\section{Takedown policy}

If you believe that this document breaches copyright please refer to the University of Manchester's Takedown Procedures [http://man.ac.uk/04Y6Bo] or contact uml.scholarlycommunications@manchester.ac.uk providing relevant details, so we can investigate your claim.

\section{OPEN ACCESS}




\section{Data Reduction for an ICT Network in the Smart Grid}

\author{
Zoya Pourmirza \\ The School of Computer Science \\ The University of Manchester \\ Manchester, UK \\ zoya.pourmirza@manchester.ac.uk
}

\author{
John M. Brooke \\ The School of Computer Science \\ The University of Manchester \\ Manchester, UK \\ John.brooke@manchester.ac.uk
}

\begin{abstract}
Intelligent electrical networks called Smart Grids incorporate information and communication technology (ICT) to service the power grid. In this paper we discuss about our proposed ICT architecture at the level of the electrical network where monitoring and control has not previously been deployed. We describe an actual project that is being implemented on the medium voltage power network of the University of Manchester, that will offer more intelligence to the current grid. Energy constraints are one of the major limitations of the ICT in the Smart Grid, especially where wireless networking is proposed. The main contribution of this paper is that we propose a data reduction algorithm suitable for Smart Grid applications which significantly improves the energy efficiency of the communication network by minimizing the communication energy cost and optimizing the network resource consumption while maintaining the integrity and quality of data.
\end{abstract}

Smart-Grid; ICT-Architecture; Energy-Efficiency; DataReduction; Neighbourhood Area Network (NAN).

\section{INTRODUCTION}

Smart Grids are intelligent electrical networks that incorporate communications and the information technology to service the generation, transmission and finally the distribution network of the power grid. The additional Information and Communication Technology (ICT) over electrical grid enables energy monitoring and control. The real-time data provided by ICT enables the identification of problems with the electrical network in advance and thus to take remedial action.

There currently exists relatively little research on the monitoring and predictive real-time system control in the Neighbourhood Area Network (NAN) of the Smart Grid. We have designed an energy efficient ICT architecture for a NAN. In this paper we will discuss an actual implementation of this design currently being installed in the sub-Grid supplying the University of Manchester that is of comparable size and complexity to urban NANs.

The wireless sensor network (WSN) supplied by the monitoring network in our ICT architecture is responsible for collecting data from the grid and transmitting these data to the control centres for providing finer grained control. These sensors are used to boost the awareness of the grid condition and asset management. Since battery technology used in the WSN has a slower development rate than both the sensor device technology and the processor technology [1], these sensors are energy constrained. If wireless sensors die, e.g. through battery rundown, we can no longer monitor their specific part of the network which may result in poor decision making. Thus, we have developed a technique to improve the energy efficiency of the WSN used in the ICT network of the Smart Grid.

The remainder of this paper is organised as follows: section 2 discusses the proposed ICT for the NAN in the Smart Grid. Section 3 presents techniques for improving energy efficiency in the monitoring network. Section 4 explains the data reduction method we have developed for Smart Grid applications. Finally section 5 summarizes our contributions and presents ideas for future work.

\section{PROPOSAL FOR AN ICT ARCHITECTURE}

Since the existing centralized structure of the control network is no longer adequate for the Smart Grid applications [2], we migrate from centralized architecture to distributed one. We have chosen a hybrid system where the wireless communication is the main communication technology; however, wired systems such as Power Line Communication (PLC) are used as a backup to enable the information flow. Moreover, to optimize the energy efficiency of the system we have developed a data reduction algorithm (section 4) that is efficient in terms of data transmission for data from the electric grid. We also analyse its dependency on the number of transmitted bits, and the speed of the compression and decompression algorithm.

Our proposed architecture is a hierarchical architecture. This architecture is the integration of six layers that cooperate to provide four functions of monitoring, data movement, data storage and control. It is designed for the future intelligent power grid, such that it is able to incorporate different control strategies at various level of the NAN.

Figure 1 shows the abstract view of our architecture first introduced in [3]. The first three layers of the architecture are in charge of sensing, measuring and relaying the data. The sensors in each of these three layers are chosen according to the data type they measure. The other three layers are responsible for computing, storing, visualising, and controlling the NAN.

The level 1 and 2 of the architecture represents the WSN. These are the sensors located at the street level, collecting data about environmental conditions. The sensors in second level 
are more powerful sensors in terms of resource capabilities such as computation ability, battery lifetime, and transmission range. They are responsible for collecting data from the first level, applying computation and transmitting the processed data to the database layer in the fifth level of the architecture. In order to evaluate the WSN of the proposed architecture, we extended the WSN query processing engine called TinyDB [4] by adding a Smart Grid component to it. Thus now we are able to simulate the environmental data, receive live data in response to the queries, and feed the received data to another source of computation to apply real-time system control.

The third level of the architecture, which is located at the substation level, is implemented by a reconfigurable real-time control and acquisition system called Compact RIO (cRIO), data storage, and a router to relay the data to the higher level. The cRIO [5] in each box is composed of the FPGA module, real-time controller, reconfigurable I/O module, and external expansion chassis. These boxes send the live electrical grid data to the fourth level.

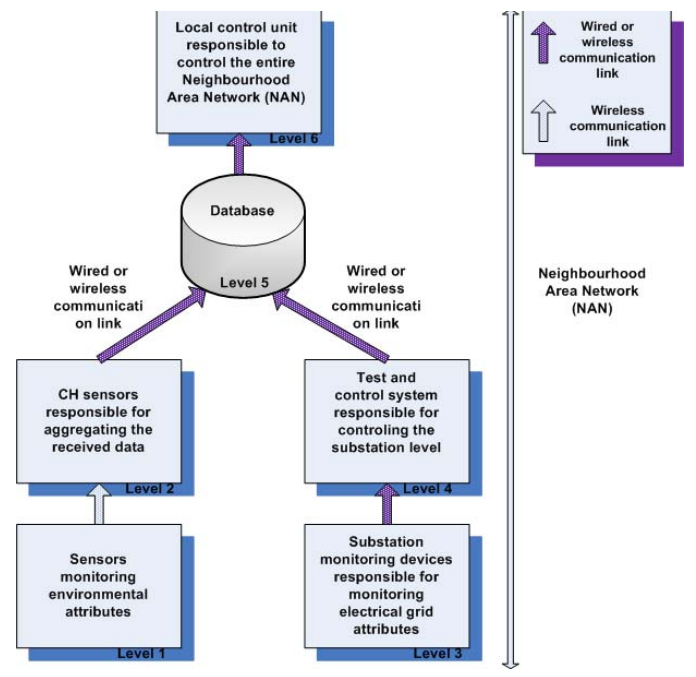

Figure 1. The ICT Architecture [6].

The fourth level of the architecture is responsible to apply control over the substation level only, and relay the received data to the fifth layer, the database level. The database stores all the data from the three levels of monitoring.

The sixth level is the local control unit, which extracts data from the database. We have developed a GIS-enhanced visualization tool for the grid operator which is located at the sixth layer [7]. This overlays information received from sensors onto a map-based view of the NAN. Based on the received data from the three monitoring layers a human operator or an automated algorithm can apply control over the whole NAN sub-Grid. In emergency situations this layer can directly send request and extract data from the sensing levels.

Each sub-Grid in the Smart Grid is composed of several such NANs. Figure 1 shows one NAN only. Several such NANs need to talk together to prevent any conflicting control decisions. In order to enable this communication another level of communication is added over the entire architecture. This added peer-to-peer architecture over the proposed hierarchical architecture will optimize the decision making in the sub-Grid and as the result make the grid more intelligent.

\section{ENERGY EFFICIENCY}

As discussed earlier the sensor network is implemented in the electrical grid to offer more intelligent to the current grid. The reason why we choose the wireless technology as means of communication between sensors and computational unit is discussed in [3]. In order to prevent poor communication and wrong decision making in the proposed architecture, our system design is targeted towards the solution of the energy constraint problem of the WSN in the Smart Grid.

The sensors used in our architecture require energy for various tasks; sensing the environment, processing the collected data, and transmitting the gathered data to a more powerful computation unit. Since the greatest amount of energy is used for the transmission rather than sensing or computation [8], it is necessary to develop a technique to decrease the transmission energy required by the whole sensor network. Therefore we can optimize the energy efficiency of our ICT architecture.

According to the first order radio model, the energy spent by the sensor for data transmission can be analysed by the following formula (ibid) and [9]:

$$
E_{T}=E_{\text {elec }} k+E_{\text {amp }} k d^{2}
$$

Where $E_{T}$ is the total energy spent for transmitting the data, $E_{\text {elec }}$ is the energy spent by the radio per bit to run the transceiver circuitry, $k$ is the number of sent bits, $E_{a m p}$ is the energy spent per bit per square unit for the transmitting amplifier, and finally $d$ is the distance between the sender sensor and the receiver node.

Formula (1) in MKS units gives values of the $E_{\text {elec }}$ and $E_{a m p}$ as $5 \times 10^{-8}$ (joule/bit) and $10^{-10}$ (joule/bit/meter ${ }^{2)}$ ) respectively. These numbers vary from sensor to senor but since $k$ and $d$ are very large compared to $\mathrm{E}_{\text {elec }}$ and $E_{a m p}$ in formula (1), these latter terms are negligible compared to $\mathrm{E}_{\mathrm{T}}$. However, the two important factors that have significant influence on the transmission energy are the transmission distance and the number of transmitting bits.

\section{A. Transmission Distance}

For a fixed number of bits, the term that controls $\mathrm{E}_{\mathrm{T}}$ is the square of distance, thus $d$ is the dominant term. Since we need to keep distance as small as possible, we have used cluster based communication rather than direct communication in the WSN of the Smart Grid. In [6] we have discussed the architecture of the WSN and have established that for a given number of sensors distributed on a rectangular there is an optimal cluster size for minimum energy consumption. For example in a grid of 100 locations the minimum is attained when the size of the clusters is 8 in the grid. This analysis also shows that the optimal shape of the clusters is a square (ibid). We also concluded that, in a network with different rectangular cluster shapes and sizes, the optimal WSN geometry is when 
the bigger clusters are located farther away, and the smaller ones close to, the base station. This is essentially a geometric effect caused by the fact that the dependence of energy required for transmission depends on the square of the distance between the receiving and sending points.

\section{B. Number of Transmitted Bits}

The second important factor in preserving the energy is the number of transmitted bits. Given that the energy consumed for transmitting one bit is equal to the energy for processing 1000 instructions [10], an important method for saving the energy is to use data reduction. The data reduction techniques can be classified into lossy and lossless methods. In the lossy reduction method the receiver generates the original data with some degree of information loss. This technique is useful for the applications that can tolerate the data loss such as video and audio compressions. Conversely in the lossless technique the receiver can generate the original data without information loss. This method is useful in applications where data loss is not acceptable, such as medical images and computer executable files [11]. Since the control protocols of the Smart Grid are still being developed, we do not yet know how much data loss is acceptable in transmission. Therefore our design requires that the whole procedure of data transmission, including compression and decompression be lossless. In the next section we have described our developed data reduction algorithm.

\section{THE DATA REDUCTION TECHNIQUE}

Data compression is the procedure of processing raw data into a condensed structure rather than its original format. One of the challenges in the data compression technique is the accuracy of the decompression algorithm while reconstructing the data. Data reduction can be classified as in-network data compression, data fusion, and data aggregation methods.

\section{A. Related Works on Data Reduction}

The data compression is used in the applications where the full data collection is required. An Example for such a technique is the Pipelined In-Network Compression (PINCO) [12]. The PINCO which uses tree based routing algorithm in the WSN assigns an aggregation node to collect all the received data in its buffer and waits for a specific duration of time to create one new data packet out of the received data. The second data reduction technique is data fusion, which is a more elegant method in comparison with the data aggregation. In this method various unreliable data will be combined to eliminate the related noise and produce a more accurate signal. The third method is data aggregation, which is useful when the goal is to reduce the communication overhead and cost. This method will reduce the message size by utilizing one of the aggregation functions such as Min, Max, Sum, and Average [13].

\section{B. Our Data Reduction Algorithm}

We work with electrical engineers implementing in a university sub-Grid at the University of Manchester. Since they are still evaluating the data we do not use fusion or aggregation. Thus, our data reduction algorithm is purely a compression method which keeps the quality and integrity of data.

\section{1) Design}

In the Smart Grid applications where the metering devices collect data with a high acquisition rate, a great degree of data correlation occurs. Taking this fact into consideration, we have developed a data reduction algorithm which discards the redundancy between each two consecutive measured values, and transmits the changing parts only. This algorithm can improve the energy efficiency of the communication network by transmitting smaller value while keeping the data integrity.

In the sender side of the proposed algorithm the collected data ready for transmission are in decimal formats. At the beginning of each round of transmission the sender will transmit the original full value of the first measured data. This full value indicates the start of each round of transmission. Frequently transmitting the original value of the measured data will reduce the risk of data loss in the communication network. If a number of measured data are missed in the transmission channel, then the receiver side will decode the received values incorrectly. In order to prevent such a data loss, our strategy is to send the full measured value on a regular basis.

To discard the redundancy between two consecutive values, the collected data (in decimal format) will be converted to binary format. Then we initiate applying exclusive OR (XOR) starting from the most significant bits. The result of XOR will be converted to decimal format before transmission. This is because our method requires some method of indicating the start of the next value and the values are of varying size as binary numbers. We send both the full values and the changes as a comma separated value (csv) stream. We determined that this extra encoding does not significantly impair the efficiency of the algorithm.

On the receiver side, the reception device will then convert the received decimal data to the binary format. The original values of the data can be generated by applying the XOR operation to the received value " $n$ " and to the reconstructed value "n-1". Figures 2 and 3 demonstrate the compression and decompression algorithms on the sender and receiver side. The pseudo codes of these algorithms are available in the author's webpage ${ }^{1}$.

\section{2) Evaluation}

Although the proposed algorithm is applicable to diverse data size and behaviour, it is most suitable for data where consecutive values vary only in the least significant bits, i.e. the rate of change of the data is slow with respect to the sending rate. The efficiency of this process, which depends on the degree of data correlation, can be described by the following formula:

Compression efficiency $=($ original data size compressed data size)/(original data size)\%.

In Figure 4 we present results of the performance of algorithm for seven diverse cases, each comprised of 17982 data values. These are the simulated data for one hour, being

\footnotetext{
${ }^{1} \mathrm{http}: / /$ personalpages.manchester.ac.uk/postgrad/zoya.pourmirza/
} 
logged approximately 5 times a second. Using the above formula our experiments reveal that in normal condition of the electrical grid we are able to achieve over $70 \%$ efficiency on average in terms of data transmission.

$\begin{aligned} & \text { The first sample of the measured data in } \\
& \text { each round is sent without compression }\end{aligned}$
\begin{tabular}{|c|c|c|l|l|}
\hline Measured value & XOR & \multicolumn{2}{|c|}{ Send value } \\
\hline Dccimal & Binary & & Binary & Dccimal \\
\hline 57 & 111001 & 111001 & 111001 & 57 \\
\hline 62 & 111110 & 000111 & 111 & 7 \\
\hline 61 & 111101 & 000011 & 11 & 3 \\
\hline 57 & 111001 & 000100 & 100 & 4 \\
\hline 63 & 111111 & 000110 & 110 & 6 \\
\hline
\end{tabular}

Figure 2. The transmitter.

\begin{tabular}{|c|r|r|c|c|}
\hline \multicolumn{2}{|c|}{ Received value } & \multicolumn{1}{c|}{ XOR } & \multicolumn{2}{c|}{ Generated value } \\
\hline Decimal & Binary & & Binary & Decimal \\
\hline 57 & 111001 & 111001 & $\mathbf{1 1 1 0 0 1}$ & 57 \\
\hline 7 & 111 & 110 & 111110 & 62 \\
\hline 3 & 11 & 01 & 111101 & 61 \\
\hline 4 & 100 & 001 & 111001 & 57 \\
\hline 6 & 110 & 111 & 111111 & 63 \\
\hline
\end{tabular}

Figure 3. The receiver.

This algorithm is efficient in terms of communication energy consumption and transmission time which depends on the number of transmitted bits. It is also efficient in terms of both the number of instructions for implementing the algorithms and the execution time which will takes less than 0.2 milliseconds for $1 \mathrm{~KB}$ of data. Saving the data in the compressed format in the DB will also save storage space, which will ease the storage constraint problem. Finally regarding the ratio of energy spent during the transmission to the energy spent during the processing, our proposed algorithm is highly energy efficient. This algorithm will use less than 100 instructions for compressing and uncompressing one bit of data, which is an order of magnitude less than the energy ratio of 1000:1 between transmission and processing.

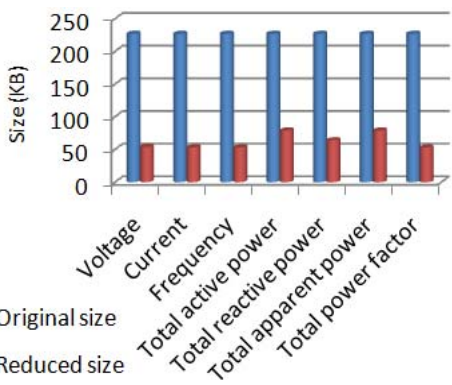

Figure 4. Comparison of original and compressed data size.

\section{CONCLUSION}

This research was undertaken to design a future proof ICT architecture for the NAN in the intelligent electrical Grid, and to evaluate the techniques for improving energy efficiency in it. Given that the major limitation of the proposed architecture lies in the fact that the metering devices are energy constrained, we have developed a practical data reduction technique. One significant contribution of our research is that the developed algorithm can improve the energy efficiency and prolong the network lifetime. Also it can provide the efficient flow of the information by reducing the data traffic and accelerating the data travelling speed. Ultimately the bottleneck caused by large volume of data can be prevented. At the end, the main value of our research is that it can deliver the information from sub-Grids below $33 \mathrm{kV}$, which remains unknown until now and convert a blind grid to a more intelligent grid.

In future, we will compare the efficiency of our proposed algorithm to other data reduction algorithms. Finally, the last stage of this research is to investigate the control strategies, and provide a pattern for, control of the Smart Grid.

\section{REFERENCES}

[1] Miao, G., et al., Cross-layer optimization for energy-efficient wireless communications: a survey. Wireless Communications and Mobile Computing, 2009. 9(4): p. 529-542.

[2] Yang, Q., J.A. Barria, and T.C. Green, Communication Infrastructures for Distributed Control of Power Distribution Networks. Industrial Informatics, IEEE Transactions on, 2011. 7(2): p. 316-327.

[3] Pourmirza, Z. and J. Brooke. AN EXPERIMENTAL COMMUNICATION ARCHITECTURE FOR MONITORING AND CONTROL OF SUBGRIDS. in SMARTGREENS 2012 - 1st International Conference on Smart Grids and Green IT Systems. 2012. Porto, Portugal: SCITEPRESS - Science and Technology Publications.

[4] Madden, S., J. Hellerstein, and W. Hong, TinyDB: In-Network Query Processing in TinyOS. 2003. p. 1-46.

[5] NI CompactRIO - Reconfigurable Control and Acquisition System. 2010 02/07/2011]; Available from: http://www.ni.com/compactrio/whatis/.

[6] Pourmirza, Z. and J.M. Brooke. The Wireless Sensor Network and Local Computational Unit in the Neighbourhood Area Network of the Smart Grid. in International Conference on Sensor Networks (SENSORNETS). 2013. Barcelona, Spain: SCITEPRESS - Science and Technology Publications.

[7] Pourmirza, Z. and J. Brooke. Monitoring and Visualising a Neighbourhood Area sub-GRID. in SMARTGREENS 2013 - 2nd International Conference on Smart Grids and Green IT Systems. 2013. Aachen, Germany: SCITEPRESS - Science and Technology Publications

[8] Heinzelman, W.R., A. Chandrakasan, and H. Balakrishnan. EnergyEfficient Communication Protocol for Wireless Microsensor Networks. in Proceedings of the 33rd Hawaii International Conference on System Sciences-Volume 8. 2000: IEEE Computer Society.

[9] Saini, S., R.S. Singh, and V.K. Gupta, Analysis of Energy Efficient Routing Protocols in Wireless Sensor Networks. International Journal of Computer Science \& Communication, 2010. 1: p. 113-118.

[10] Sacaleanu, D.I., et al. Compression scheme for increasing the lifetime of wireless intelligent sensor networks. in Signal Processing Conference (EUSIPCO), 2012 Proceedings of the 20th European. 2012.

[11] KODITUWAKKU, S.R. and U.S. AMARASINGHE, COMPARISON OF LOSSLESS DATA COMPRESSION ALGORITHMS FOR TEXT $D A T A$. Indian Journal of Computer Science and Engineering, 2011. 1(4): p. 416-425.

[12] Arici, T., et al. PINCO: a pipelined in-network compression scheme for data collection in wireless sensor networks. in Computer Communications and Networks, 2003. ICCCN 2003. Proceedings. The 12th International Conference on. 2003.

[13] Tan, H.O., I. Korpeoglu, and I. Stojmenovic, A Distributed and Dynamic Data Gathering Protocol for Sensor Networks, in Proceedings of the 21 st International Conference on Advanced Networking and Applications. 2007, IEEE Computer Society. p. 220-227. 